\title{
Clinical laboratory test-wide association scan of polygenic scores identifies biomarkers of complex disease
}

Jessica K. Dennis ${ }^{1,2,3 \dagger}$, Julia M. Sealock ${ }^{1,2 \dagger}$, Peter Straub ${ }^{1,2}$, Younga H. Lee ${ }^{4,5,6}$, Donald Hucks ${ }^{1,2}$, Ky $^{\prime}$ Era Actkins $^{1,2,7}$, Annika Faucon ${ }^{1,2}$, Yen-Chen Anne Feng ${ }^{4,6,8}$, Tian Ge ${ }^{4,5,6}$, Slavina B. Goleva ${ }^{1,2,9}$, Maria Niarchou ${ }^{1,2}, K^{\prime}$ ritika Singh ${ }^{1,2}$, Theodore Morley ${ }^{1,2}$, Jordan W. Smoller ${ }^{4,5,6}$, Douglas M. Ruderfer ${ }^{1,2,10,11}$, Jonathan D. Mosley ${ }^{11}$, Guanhua Chen ${ }^{12}$ and

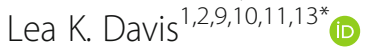

\begin{abstract}
Background: Clinical laboratory (lab) tests are used in clinical practice to diagnose, treat, and monitor disease conditions. Test results are stored in electronic health records (EHRs), and a growing number of EHRs are linked to patient DNA, offering unprecedented opportunities to query relationships between genetic risk for complex disease and quantitative physiological measurements collected on large populations.

Methods: A total of 3075 quantitative lab tests were extracted from Vanderbilt University Medical Center's (VUMC) EHR system and cleaned for population-level analysis according to our QualityLab protocol. Lab values extracted from BioVU were compared with previous population studies using heritability and genetic correlation analyses. We then tested the hypothesis that polygenic risk scores for biomarkers and complex disease are associated with biomarkers of disease extracted from the EHR. In a proof of concept analyses, we focused on lipids and coronary artery disease (CAD). We cleaned lab traits extracted from the EHR performed lab-wide association scans (LabWAS) of the lipids and CAD polygenic risk scores across 315 heritable lab tests then replicated the pipeline and analyses in the Massachusetts General Brigham Biobank.

Results: Heritability estimates of lipid values (after cleaning with QualityLab) were comparable to previous reports and polygenic scores for lipids were strongly associated with their referent lipid in a LabWAS. LabWAS of the polygenic score for CAD recapitulated canonical heart disease biomarker profiles including decreased HDL, increased pre-medication LDL, triglycerides, blood glucose, and glycated hemoglobin (HgbA1C) in European and African descent populations. Notably, many of these associations remained even after adjusting for the presence of cardiovascular disease and were replicated in the MGBB.

(Continued on next page)
\end{abstract}

\footnotetext{
* Correspondence: lea.k.davis@gmail.com

${ }^{\dagger}$ Jessica K. Dennis and Julia M. Sealock contributed equally to this work.

'Division of Genetic Medicine, Department of Medicine, Vanderbilt University Medical Center, Nashville, TN 37232, USA

${ }^{2}$ Vanderbilt Genetics Institute, Vanderbilt University Medical Center, Nashville,

TN 37232, USA

Full list of author information is available at the end of the article
}

(c) The Author(s). 2021 Open Access This article is licensed under a Creative Commons Attribution 4.0 International License, which permits use, sharing, adaptation, distribution and reproduction in any medium or format, as long as you give appropriate credit to the original author(s) and the source, provide a link to the Creative Commons licence, and indicate if changes were made. The images or other third party material in this article are included in the article's Creative Commons licence, unless indicated otherwise in a credit line to the material. If material is not included in the article's Creative Commons licence and your intended use is not permitted by statutory regulation or exceeds the permitted use, you will need to obtain permission directly from the copyright holder. To view a copy of this licence, visit http://creativecommons.org/licenses/by/4.0/ The Creative Commons Public Domain Dedication waiver (http://creativecommons.org/publicdomain/zero/1.0/) applies to the data made available in this article, unless otherwise stated in a credit line to the data. 
(Continued from previous page)

Conclusions: Polygenic risk scores can be used to identify biomarkers of complex disease in large-scale EHR-based genomic analyses, providing new avenues for discovery of novel biomarkers and deeper understanding of disease trajectories in pre-symptomatic individuals. We present two methods and associated software, QualityLab and LabWAS, to clean and analyze EHR labs at scale and perform a Lab-Wide Association Scan.

Keywords: Electronic health records, Population genetics, Genetic epidemiology, Biomarkers

\section{Background}

The overarching goal of this study was to determine whether laboratory (lab) test results collected in a hospital and outpatient setting could be mined against polygenic scores (PGS) to identify known and novel biomarker associations for complex disease. Lab test results are essential to routine clinical care. These targeted biochemical measurements facilitate disease diagnosis and influence health care delivery. Clinical lab values are also monitored as mediators of disease risk and are targeted by interventions to reduce disease incidence (e.g., cholesterol-lowering medication to reduce the risk of heart disease). Lab test results in the electronic health record (EHR) are a vast and growing resource for novel biomarker discovery, especially as EHRs are increasingly linked to patient DNA samples (e.g., the eMERGE consortium (https://emerge.mc.vanderbilt.edu)), the All of Us Program (https://allofus.nih.gov), and the Million Veteran's Program (https://www.research.va.gov/mvp/)). Genetic studies of EHR-based labs could reveal novel biomarker-disease or biomarker-gene associations, which in turn could lead to better understanding of biological processes in disease, improved diagnostic algorithms, and new therapeutic targets.

Despite their potential, however, EHR-based labs have been used in only a handful of prior genetic studies [1-5], and none have systematically interrogated an extended collection of EHR-based lab values. Barriers to studying EHR-based labs include uneven data quality, and challenges inherent to analyzing and interpreting highdimensional health care data. Data entry errors exist, resulting in implausible recorded values [6], some labs have different units and reference ranges over time, and many individuals have multiple observations of different lab tests, each measured at varying times relative to diagnoses and treatment [7]. Moreover, previous studies demonstrate that while $99 \%$ of lab results are accurately transmitted from the testing laboratory to the EHR, only $70 \%$ of test results contain all required reporting elements, and only $91 \%$ of results are appropriately formatted [8]. Thus, while these data represent real clinical care and may accelerate translational research, there is little precedent for their analysis and interpretation in genetic studies.

To address these challenges, we present a highthroughput framework for genetic analysis of EHR-derived lab data. We have developed two methods: the QualityLab pipeline to clean, standardize, and visualize lab data and the Lab-Wide Association Scan (LabWAS) pipeline to scan for associations between any variable of interest (genetic or otherwise) and the cleaned EHR labs. The LabWAS method is similar to the Phenome-Wide Association Scan (PheWAS) which scans for association between an exposure variable (typically, a genetic risk factor) and many phenotypes [9]. The PheWAS method has replicated many known gene-disease associations [10] and has identified novel pleiotropic genetic effects [11], opportunities for drug repurposing, and unintended drug consequences [12]. QualityLab builds off the success of previous measurement quality control methods, such as CLARITE [13]. While, CLARITE focuses on minimal cleaning of survey data, QualityLab conducts extensive cleaning of quantitative lab measurements derived from EHRs.

We hypothesized that EHR-based lab values could be used to identify known and novel relationships between genetics, biomarkers, and disease. We deployed our framework in the Vanderbilt University Medical Center (VUMC) EHR and linked biobank, BioVU, and replicated it in an independent biobank, Massachusetts General Brigham Biobank. We focused on genetic analysis of blood values of high-density lipoprotein cholesterol (HDL), low-density lipoprotein cholesterol (LDL), and triglycerides (TG) and on coronary artery disease (CAD) as proof-of-principle examples to test the association between PGS for CAD and known biomarkers of disease (LDL, HDL, and TG) using the QualityLab and LabWAS methods across populations. We show that EHR-derived lipids values are genetically similar to those in population-based studies and that PGS for lipids robustly associate with their respective lab in a LabWAS. Additionally, our LabWAS revealed that PGS for CAD associated with known lipid biomarkers, even in individuals without a history of CAD, and with potentially novel immune biomarkers.

\section{Methods}

Study sample

Our primary analysis was performed at VUMC which is a tertiary care center providing inpatient and outpatient care in Nashville, TN. The VUMC EHR was established in 1994 and includes data on billing codes from the 
International Classification of Diseases, 9th and 10th editions (ICD-9 and ICD-10), Current Procedural Terminology (CPT) codes, laboratory values, reports, and clinical documentation. The de-identified mirror of the EHR, known as the Synthetic Derivative, includes patient records on more than 2.8 million individuals. In 2007, VUMC launched a biobank, BioVU, and the BioVU Consent form is provided to patients in the outpatient clinic environments at VUMC. The form states policies on data sharing and privacy and, upon consent, makes any blood leftover from clinical care eligible for BioVU banking [14]. The VUMC Institutional Review Board oversees BioVU and approved this project.

\section{Genotyping and quality control}

We obtained genotype information on 94,474 BioVU individuals of different ancestral and racial backgrounds genotyped on the Illumina MEGA ${ }^{\mathrm{EX}}$ array. Using PLINK v1.9 [15], genotypes were filtered for SNP and individual call rates, sex discrepancies, and excessive heterozygosity (Additional file 1). We selected individuals of European or African ancestry using principal component analysis implemented in Eigenstrat [16, 17] and confirmed the absence of genotyping batch effects through logistic regression with "batch" as the phenotype. Imputation was completed using the Michigan Imputation Server [18] using the Haplotype Reference Consortium (HRC) reference panel. SNPs were then filtered for SNP imputation quality $\left(R^{2}>0.3\right)$ and converted to hard calls. We restricted to autosomal SNPs, filtered SNPs with minor allele frequency $>0.01$, or with allele frequencies that differed by more than 10\% from the 1000 Genomes Project phase 3 CEU or ASW set respectively [19], and HardyWeinberg Equilibrium $\left(p>1 \times 10^{-10}\right)$. The resulting dataset contained 6,303,629 SNPs on 72,824 individuals of European genetic ancestry and 12,798,111 SNPs on 15,283 individuals of African genetic ancestry.

\section{QualityLab pipeline}

In parallel with the BioVU genotyping project, we extracted data on all lab tests collected in the routine clinical care of $1,521,125$ VUMC patients, amounting to 275,991,157 observations across 11,061 lab tests (Fig. 1a). Of these lab tests, 5028 were reported in non-numeric values and 1618 had only been administered to one patient, leaving 4415 quantitative lab tests for further cleaning. Some lab tests had observations recorded in different units (e.g., Selenium reported in both $\mathrm{mcg} / \mathrm{L}$ and $\mu \mathrm{g} / \mathrm{L}$ ); thus, we restricted to lab tests for which at least $70 \%$ of the observations were measured in the same unit and required that each lab have at least 100 patients and at least 1000 numeric observations, for a total of 939 labs retained for further analysis.
For each of these 939 labs, we applied lab-specific quality control filters (Fig. 1b). First, we filtered infinite and non-numeric values, as well as observations outside of 4 standard deviations from the overall sample mean, indicative of biologically implausible values due to technical or recording errors, monogenic disorders, or extreme environmental influence. We calculated the median lab value for each patient and extracted the patient's age at median lab value. For patients in whom we had to calculate the median lab value (e.g., those with an even number of observations), we defined the age at median lab value as the mid-point of the patient's ages at the two lab values used to calculate the median lab value.

The analyses presented in this manuscript use the QualityLab dataset constructed from pediatric and adult observations, in both sexes, in patients of all races (Fig. 1b). In downstream genetic analyses, however, we restrict to participants of European or African genetic ancestry and match the ancestry of the participants in the discovery GWAS used for the training the PGS.

The QualityLab pipeline also provides user with the option to stratify data (Fig. 1b), by age at observation, sex, and EHR-recorded race, for a total of 72 different data subsets. The QualityLab pipeline generates summary statistics and plots for each strata (e.g., mean, maximum, and minimum of the median lab value; Additional file 2: Table S1; Additional file 3: Fig. S1), and returns two versions of the data for downstream analyses. The first is a table of median lab values and age at median lab value for each individual. The second is an inverse normal quantile transformation (INT) of the median lab value data, to account for skewness and non-normality [20, 21]. Importantly, the choice of quality control thresholds is completely in the control of the user. The choices made here reflect the goals of this study which focus on the central tendencies of large populations. However, the outlier thresholds and normalization methods employed here would not be appropriate in a study of rare, potentially pathogenic, variation where large genetic effects and extreme phenotypes may be expected.

\section{Lab heritability and GWAS analyses}

Prior to calculating SNP-based heritability $\left(\mathrm{h}_{\mathrm{SNP}}^{2}\right)$, we first calculated pairwise relatedness in the BioVU genotyped sample and removed one related individual from pairs with pi-hat greater than 0.05 . This stringent threshold was chosen based on prior experience and previously published best practices in the application of restricted maximum likelihood (REML) approaches to

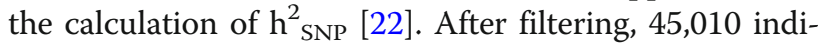
viduals of European genetic ancestry (Fig. 1a) remained. We then used the genome-wide complex trait analysis (GCTA) package (version 1.92.4) [23] to create a pairwise genetic relationship matrix for all individuals, and 
a

a

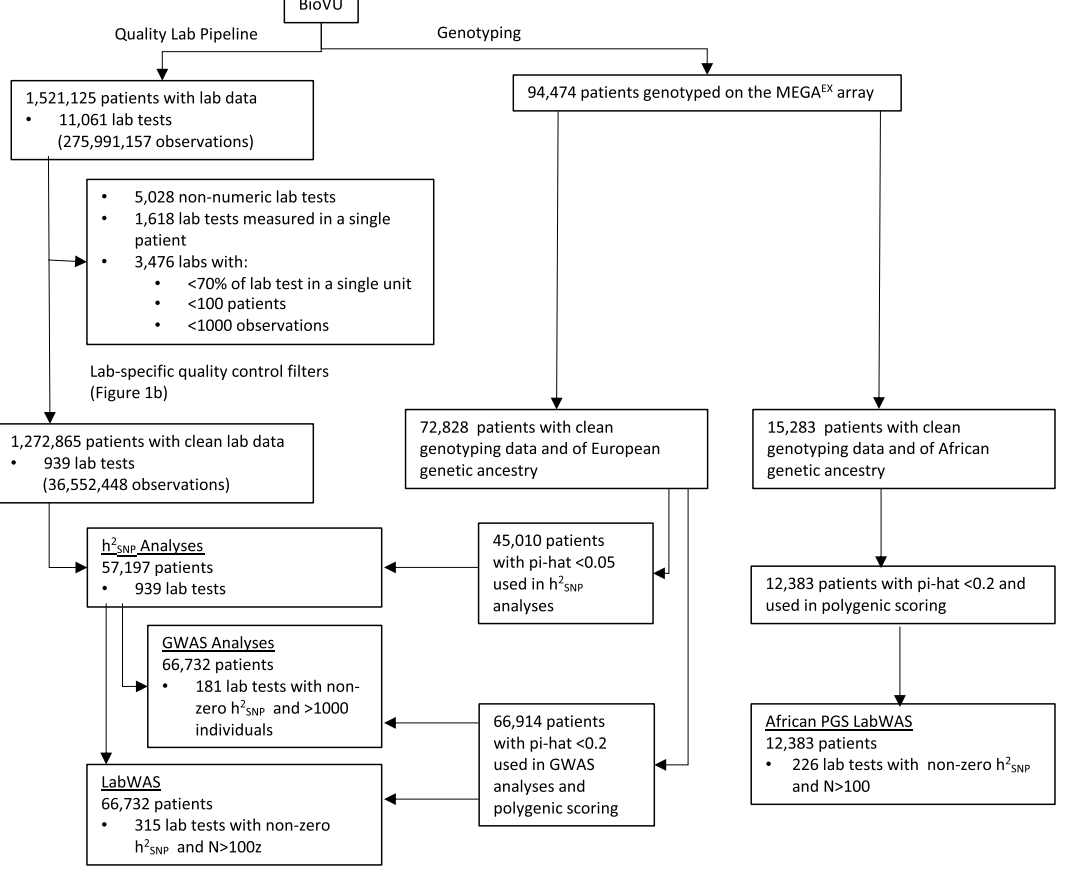

b

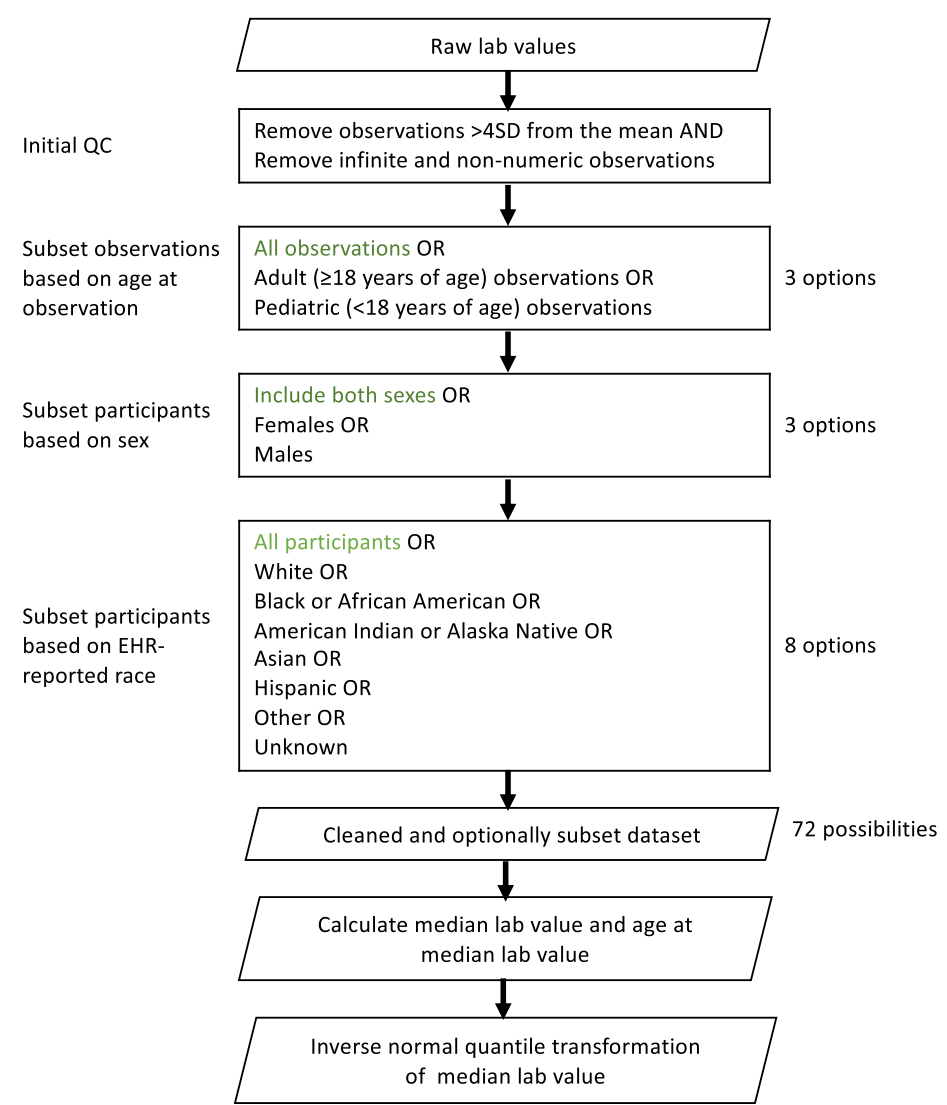

Fig. 1 (See legend on next page.) 
(See figure on previous page.)

Fig. 1 Selection of BioVU patients and datasets for different analyses presented in this manuscript. a BioVU patients were selected in parallel for clinical laboratory (lab) test cleaning and for genotyping. b Lab-specific quality control filters and subsetting were applied to the 939 lab tests in the 94,474 patients with clean lab data. Parallelograms denote input and output datasets. Options highlighted in green were selected for the proof-of-principle analyses of blood-based lipid lab values

heritabilities were calculated using REML methods. We used the median, INT-transformed lab values from the QualityLab pipeline, and of the 481 analyzed labs, 335 demonstrated non-zero heritability. For GWAS analyses, we used a less stringent relatedness filter appropriate to GWAS (pi-hat >0.2) [24] resulting in a total available sample of 66,732 European descent individuals. Next, we subset to the heritable labs with at least 1000 individuals $(n=181)$ and performed GWAS of the median, INTtransformed lab values using fastGWA [25] (Fig. 1a). All $\mathrm{h}^{2}$ SNP and GWAS analyses included covariates for sex, cubic splines (knots $=4$ ) of median age across the medical record (to control for non-linear effects of age), and the top 10 principal components of ancestry.

\section{Heritability and GWAS analyses of lipids}

We benchmarked our lipid $\mathrm{h}^{2}$ SNP estimates against those from two external datasets, the Global Lipids Genetics Consortium (GLGC) [26] and the Million Veterans Program (MVP). GLGC and MVP estimates of $h^{2}{ }_{S N P}$ for HDL, LDL, and TG were calculated from GWAS summary statistics using LDSC [27]. We computed $\mathrm{h}_{\text {SNP }}^{2}$ in BioVU using Linkage Disequilibrium Score regression (LDSC) applied to our fastGWA summary statistics for HDL, LDL, and TG (Additional file 3: Fig. S2). However, because LDSC can underestimate $h_{\text {SNP }}^{2}$ [28], we also calculated $\mathrm{h}_{\text {SNP }}^{2}$ using GCTA. In addition to these $\mathrm{h}^{2}{ }_{\mathrm{SNP}}$ comparisons, we calculated the genetic correlations $\left(r_{\mathrm{g}}\right)$ between the BioVU lipid GWASs and the GLGC and MVP lipid GWASs using LDSC and the pre-computed European LD scores from 1000 Genomes Phase 3 European data [29]. We also calculated genetic correlations using a new method, high-definition likelihood [30], which fully accounts for linkage disequilibrium across the genome and is more suitable for traits with lower heritability than LDSC. In sensitivity analyses, we repeated genetic correlations of LDL after controlling the BioVU GWASs for coronary atherosclerosis or diabetes diagnoses, defined as phecodes 411, "Ischemic heart disease," and 249, "Secondary diabetes mellitus" (Additional file 1).

To validate EHR-based lipid values, we tested the robustness of HDL, LDL, and TG $\mathrm{h}^{2}{ }_{\mathrm{SNP}}$ estimates to different lab value and patient filters. First, we excluded lipid measurements that occurred after the first mention of lipid-altering mediation in the EHR (Additional file 1) and re-calculated each patient's pre-medication median values of HDL, LDL, and TG. Second, we excluded patients with a diagnosis of CAD, defined by the phecode 411 (Additional file 1).

\section{LabWAS pipeline}

LabWAS uses the median, INT-transformed lab values from the QualityLab pipeline in a linear regression to determine the association with an input variable, adjusting for covariates. In these analyses, a primary goal of the LabWAS was to test common population genetic variation (e.g., PGS) for association with common population variation in lab values. We therefore only included the 335 labs with

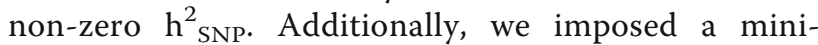
mum sample size requirement of 100 for a lab to be included in the LabWAS analysis, bringing the number of labs tested in each scan to 315 in the European ancestry set and 226 in the African ancestry set.

\section{Polygenic scoring}

Prior to polygenic scoring, we randomly removed one related individual from pairs with pi-hat greater than 0.2 , leaving 66,732 individuals of European genetic ancestry and 12,383 individuals of African genetic ancestry. (Fig. 1a). We generated lipids PGS for these individuals using PRS-CS [31] with weights derived from the transethnic MVP lipid GWAS summary statistics [4]. PGS for CAD (CAD ${ }_{\text {PGS }}$ ) was calculated using SNP weights from CARDIoGRAMplusC4D GWAS summary statistics [32] using PRS-CS. Because the majority of the MVP transethnic sample was European, linkage disequilibrium was modeled using the pre-calculated European panel. PRSCS is a recently developed Bayesian polygenic prediction method that imposes continuous shrinkage priors on SNP effect sizes (Polygenic Risk Score - Continuous Shrinkage) [31]. These priors can be represented as global-local scale mixtures of normals which allow the model to flexibly adapt to differing genetic architectures and provide substantial computational advantages. The shrinkage parameter was automatically learnt from the data (i.e., using PRS-CS-auto). SNP effect estimates were obtained from GWAS summary statistics and the score was calculated using a linkage disequilibrium reference panel from 503 European samples in the 1000 Genomes Project phase 3 [19]. Although PRS-CS outperformed other polygenic scoring methods across a range of traits in previous experiments, its superiority may not hold across all genetic architectures [31]. We therefore also generated PGS for the European sample using PRSice-2 
[33] (Additional file 1) and have automated a pipeline to generate scores across both methods. PGS were scaled to have a mean of zero and SD of one before testing for association with any outcome variables. We validated each score by testing the proportion of trait variability explained by the PGS, controlling for sex, cubic splines of median age (4 knots) across the medical record, and the top 10 principal components to adjust for genetic ancestry (Additional file 3: Fig. S3).

\section{LabWAS of polygenic scores}

PGS for LDL (PGS $\left.\mathrm{PDL}_{\mathrm{L}}\right)$, HDL $\left(\mathrm{PGS}_{\mathrm{HDL}}\right)$, and TG $\left(\mathrm{PGS}_{\mathrm{TG}}\right)$ were calculated in BioVU participants using PRS-CS and applying SNP weights from the MVP GWAS summary statistics. We then ran LabWAS of $\mathrm{PGS}_{\mathrm{LDL}}, \mathrm{PGS}_{\mathrm{HDL}}$, and $\mathrm{PGS}_{\mathrm{TG}}$ to test whether lipid labs were robustly associated with the genetic scores to which they corresponded. Next, a PGS for CAD $\left(C A D_{\text {PGS }}\right)$ was calculated using SNP weights from CARDIoGRAMplusC4D GWAS summary statistics [32] and a LabWAS of PGS $\mathrm{PAD}_{\mathrm{C}}$ to test whether the score could identify lab traits associated with genetic risk for $\mathrm{CAD}$, before and after controlling for a CAD diagnosis (Additional file 1). Each LabWAS was controlled for sex, cubic splines of median age across the medical record, and the top 10 principal components of ancestry. Results are reported as effect estimates and their $95 \%$ confidence intervals per SD increase in the PGS. The Bonferronicorrected threshold for statistical significance across all tested labs was $3.97 \times 10^{-5}(0.05 /(315 \times 4))$.

\section{Replication in Massachusetts General Brigham Biobank}

We next sought to replicate the associations between lipids PGS and referent lipids as well as the significant associations with $C_{A D} D_{\text {GGS }}$ in an external biobank. The MGBB, previously the Partners Biobank, is an ongoing virtual cohort study of patients across the Partners HealthCare hospital system (including Brigham and Women's Hospital, Massachusetts General Hospital, and other affiliated hospitals), which provides a large-scale resource of linked longitudinal electronic health records (EHR) data, genomic data, and self-reported survey data [34]. All patients provided informed consent before enrollment, and all study procedures were approved by the Partners HealthCare Institutional Review Board.

Lab values were extracted from EHRs and cleaned using QualityLab, resulting in 759 labs for analysis. The median value for each lab trait for each individual was selected and inverse normalized. Lab heritabilities were calculated using REML in GCTA. Of 759 labs that passed QualityLab, 241 demonstrated measurable heritability and included a sample size of at least 100 individuals.

Polygenic scores for HDL, LDL, TG, and CAD were calculated on individuals of European descent in MGBB
( $n=25,698)$ using the same criteria as BioVU. Lipids and CAD polygenic scores were associated with each of 234 labs using LabWAS. Lastly, the associations between $\mathrm{CAD}_{\mathrm{PGS}}$ and lab traits were controlled for CAD diagnosis, defined by phecode 411 ( $N$ cases $=1094, N$ controls $=20,405)$. All associations were controlled for sex, top 10 principal components, and the first two splines of median age across the medical record.

\section{Results}

\section{QualityLab pipeline}

A total of 94,474 BioVU patients with clean lab data, of whom 66,732 were also of European genetic ancestry were included in the PGS LabWAS analyses (Fig. 1a). These 66,732 patients had data on 939 labs, containing $30,421,498$ observations. The median number of unique lab tests per patient was 44 , and the median number of lab observations per patient was 201. Slightly more than half of the BioVU patients in the sample were female (55.6\%), and the average median age across the EHR was 52.0 years. These BioVU participants included 10,015 CAD cases and 49,702 CAD controls. In the African ancestry sample, 12,383 patients had data on 925 labs, containing 5,367,062 observations. More than half the patients were female $(61.6 \%)$ and the average median age was 38.5 years. The median number of unique lab tests per patient was 41 , and the median number of lab observations per patient was 150 (Additional file 1; Additional file 2: Table S3). Distributions of lipids levels by genetic ancestry are shown in Additional file 3: Fig. S4.

\section{Heritability and GWAS analyses}

Out of 939 clean lab traits, 335 demonstrated non-zero $\mathrm{h}_{\text {SNP }}^{2}$ and the point estimates ranged from $2 \times 10^{-6}$ to 0.98. (Additional file 2: Table S4, Additional file 3: Fig. S5). As a resource for the community, the GWAS summary statistics for the labs with calculable heritability and a minimum sample size of 1000 individuals $(n=181)$ are available in the GWAS Catalog (Study Number: GCP000091; accession numbers GCST90012603 - GCST90012784; accession numbers are listed in Additional file 2: Table S22).

\section{Heritability and GWAS analyses of lipids}

The $h_{\text {SNP }}^{2}$ estimates in BioVU were robust to removing post-medication observations, and to removing CAD cases. The number of participants included in these analyses, however, was smaller, and so the standard errors of these $\mathrm{h}_{\text {SNP }}^{2}$ estimates were larger (Fig. 2a; Additional file 2: Table S5). Both GCTA and LDSC gave similar estimates of $\mathrm{h}_{\text {SNP }}^{2}$ in BioVU (Fig. 2b), and the LDSC estimates in BioVU were comparable to those in the GLGC and MVP for all lipids. 

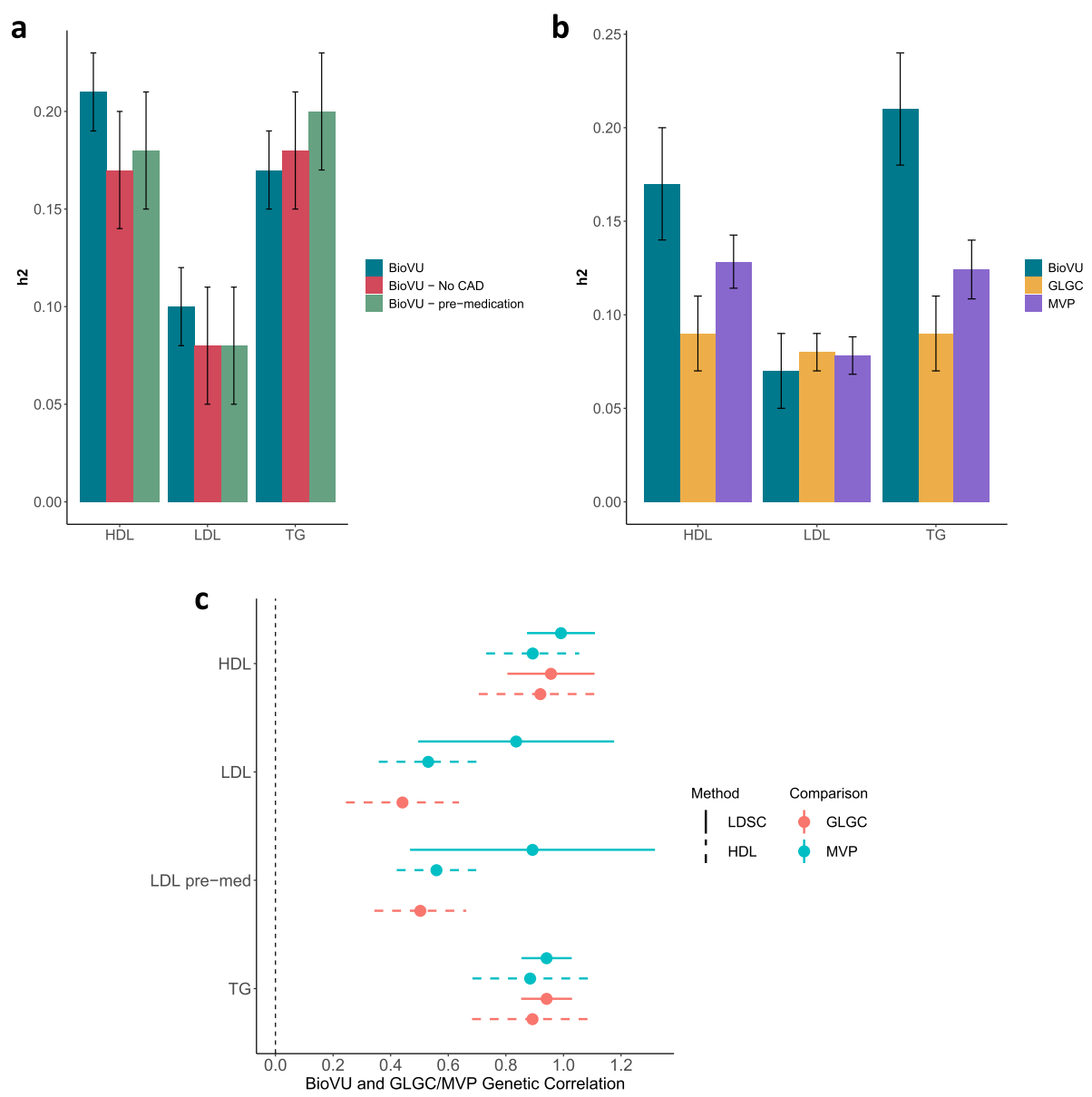

Fig. 2 Heritability and GWAS analyses of lipids. a Estimates of heritability computed by GCTA in BioVU patients were robust to excluding individuals with a diagnosis of CAD and to removing post-medication observations. b Estimates of heritability computed using GWAS summary statistics and LDSC were comparable across BioVU and the Global Lipids Genetic Consortium (GLGC) and Million Veteran's Project (MVP) samples. c Genetic correlations between lipid levels in BioVU and the Global Lipids Genetic Consortium (GLGC) or Million Veteran's Program (MVP) calculated using LDSC or high-definition likelihood (HDL). Stars denote statistically significant correlations

Genetic correlation between BioVU and GLGC summary statistics was strong for HDL (LDSC: $\mathrm{rg}=0.96$, $\mathrm{SE}=0.08, p$ value $=2.69 \times 10^{-35}$, high-definition likelihood: $\mathrm{rg}=0.92, \mathrm{SE}=0.11, p$ value $=3.25 \times 10^{-17}$ ) and $\mathrm{TG}$ (LDSC: $\mathrm{rg}=0.94, \mathrm{SE}=0.05, p$ value $=5.86 \times 10^{-97}$, highdefinition likelihood: $\mathrm{rg}=0.89, \mathrm{SE}=0.11, p$ value $=7.69 \times$ $10^{-17}$ ). When comparing BioVU and MVP, the correlations for HDL (LDSC: $\mathrm{rg}=0.99, p$ value $=7.51 \times 10^{-61}$, highdefinition likelihood: $\mathrm{rg}=0.89, \mathrm{SE}=0.08, p$ value $=2.24 \times$ $10^{-27}$ ) and TG (LDSC: $\mathrm{rg}=0.94, p$ value $=2.28 \times 10^{-99}$, high-definition likelihood: $\mathrm{rg}=0.88, \mathrm{SE}=0.10, p$ value $=$ $4.84 \times 10^{-18}$ ) were nearly perfect. The LDL and LDL premedication genetic correlations between GLGC and BioVU were not calculable using LDSC due to low heritability. Using high-definition likelihood, GLGC LDL levels were significantly correlated when median LDL values across the entire EHR $\left(\mathrm{rg}=0.44, \mathrm{SE}=0.10, p\right.$ value $\left.=1.08 \times 10^{-5}\right)$ and median pre-medication LDL values $(\mathrm{rg}=0.50, \mathrm{SE}=0.08, p$ value $\left.=6.38 \times 10^{-10}\right)$. The comparison between BioVU and
MVP showed a stronger correlation for LDL (LDSC: $\mathrm{rg}=$ $0.84, \mathrm{SE}=0.17, p$ value $=1.47 \times 10^{-6}$; high-definition likelihood: $\mathrm{rg}=0.53, \mathrm{SE}=0.09, p$ value $=1.52 \times 10^{-11}$ ). The genetic correlation with MVP increased when we restricted to pre-medication values of LDL in BioVU (LDSC: $\mathrm{rg}=$ $0.89, \mathrm{SE}=0.22, p$ value $=2.90 \times 10^{-5}$; high-definition likelihood: $\mathrm{rg}=0.56, \mathrm{SE}=0.07, p$ value $=2.06 \times 10^{-15}$ ) (Fig. $2 \mathrm{c}$ ) and increased further when we controlled for coronary atherosclerosis and diabetes diagnoses (GLGC, high-definition likelihood: $\mathrm{rg}=0.57, \mathrm{SE}=0.09, p$ value $=8.88 \times 10^{-9}$, MVP, LDSC: $\mathrm{rg}=1.00, \mathrm{SE}=0.34, p$ value $=0.004)(\mathrm{MVP}$, highdefinition likelihood: $\mathrm{rg}=0.55, \mathrm{SE}=0.09, p$ value $=1.50 \times$ $10^{-8}$ ) (Additional file 3: Fig. S6).

\section{LabWAS of polygenic scores for lipids}

A LabWAS of HDL PGS $_{\text {in }}$ the European sample was associated with levels of several metabolic markers (Fig. 3a, Additional file 2: Table S6), including increased HDL $\left(p\right.$ value $<2.23 \times 10^{-308}$, beta $\left.=0.31\right)$, decreased TG 
$\left(p\right.$ value $=2.06 \times 10^{-171}$, beta $\left.=-0.16\right)$, decreased total cholesterol to HDL ratio ( $p$ value $=2.54 \times 10^{-44}$, beta $=-$ 0.22 ), increased total blood cholesterol $(p$ value $=2.51 \times$ $10^{-37}$, beta $\left.=0.07\right)$, and decreased blood glucose $(p$ value $=$ $4.62 \times 10^{-32}$, beta $\left.=-0.04\right)$, decreased blood urea nitrogen
( $p$ value $=1.48 \times 10^{-15}$, beta $\left.=-0.03\right)$, decreased glycated hemoglobin $\left(p\right.$ value $=1.52 \times 10^{-12}$, beta $\left.=-0.05\right)$, decreased bedside glucose $\left(p\right.$ value $=1.03 \times 10^{-11}$, beta $=-$ $0.07)$, and decreased whole blood glucose $(p$ value $=2.49 \times$ $10^{-5}$, beta $\left.=-0.03\right)$. HDL $L_{\mathrm{PGS}}$ was also associated with four
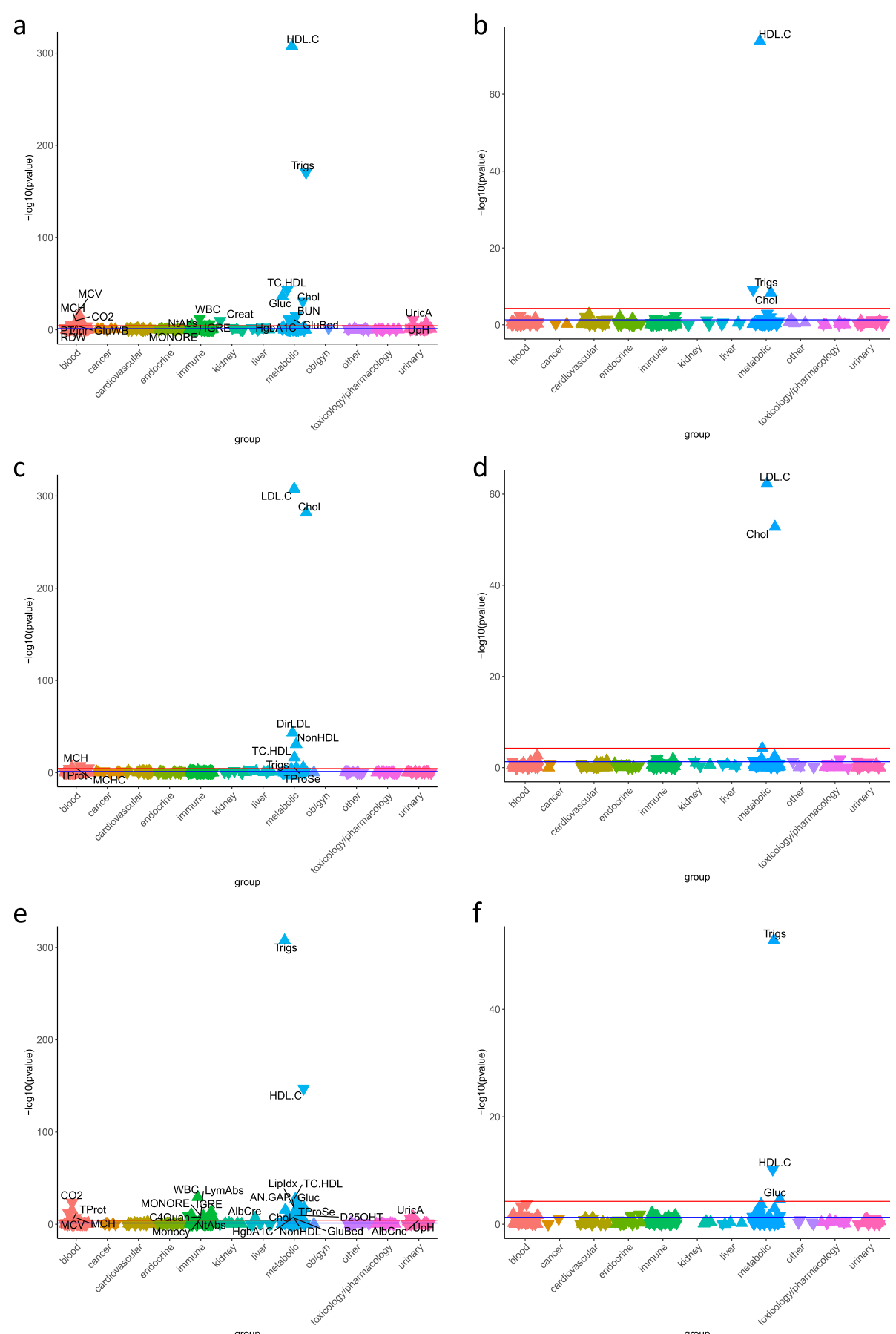

Fig. 3 LabWAS of PGS ${ }_{H D L}$ in $\mathbf{a}$ individuals of European ancestry (EA) and $\mathbf{b}$ individuals of African ancestry (AA), LabWAS of PGS $A A$, and LabWAS of PGSTG in $\mathbf{e} E A$ and $\mathbf{f}$ AA. The red line indicates the Bonferroni threshold for statistical significance and the blue line indicates a $p$ value of 0.05 . Upward triangles indicate that the PGS is associated with increased levels of the lab, while downward triangles indicate an association with reduced levels of the lab 
immune labs, white blood cell count ( $p$ value $=6.14 \times 10^{-13}$, beta $=-0.03)$, absolute neutrophil count $(p$ value $=5.69 \times$ $10^{-7}$, beta $\left.=-0.03\right)$, immature granulocytes $(p$ value $=$ $7.86 \times 10^{-6}$, beta $=-0.02$ ), and monocyte to leukocyte ratio $\left(p\right.$ value $=9.13 \times 10^{-6}$, beta $\left.=0.02\right)$. Five blood biomarkers associated with $\mathrm{HDL}_{\mathrm{PGS}}$, mean corpuscular volume $(p$ value $=3.48 \times 10^{-17}$, beta $\left.=0.03\right)$, blood carbon dioxide $\left(p\right.$ value $=6.69 \times 10^{-11}$, beta $\left.=0.02\right)$, mean corpuscular hemoglobin $\left(p\right.$ value $=9.53 \times 10^{-10}$, beta $\left.=0.02\right)$, international normalized ratio $\left(p\right.$ value $=1.31 \times 10^{-6}$, beta $=$ $-0.03)$, and red blood cell distribution width ( $p$ value $=2.21 \times 10^{-5}$, beta $\left.=-0.02\right)$. Finally, three other labs associated with $\mathrm{HDL}_{\mathrm{PGS}}$, urate $(p$ value $=1.13 \times$ $10^{-11}$, beta $\left.=-0.07\right)$, creatinine $\left(p\right.$ value $=1.42 \times 10^{-10}$, beta $=-0.02)$, and urine $\mathrm{pH}\left(p\right.$ value $=2.22 \times 10^{-8}$, beta $=0.02)$. In the African ancestry group, HDLPGS significantly associated with increased HDL ( $p$ value $=$ $1.38 \times 10^{-74}$, beta $\left.=0.23\right)$, decreased triglycerides $(p$ value $=6.72 \times 10^{-10}$, beta $\left.=-0.08\right)$, and increased total cholesterol $\left(p\right.$ value $=4.81 \times 10^{-9}$, beta $\left.=0.08\right)($ Fig. $3 \mathrm{~b}$, Additional file 2: Table S7).

The LabWAS of LDL four lipid labs (Fig. 3c, Additional file 2: Table S8). The most significant association was increased calculated LDL ( $p$ value $<2.23 \times 10^{-308}$, beta $=0.24$ ), followed by increased total blood cholesterol $\left(p\right.$ value $=1.30 \times 10^{-282}$, beta $=0.20)$, increased directly measured LDL ( $p$ value $=$ $3.79 \times 10^{-44}$, beta $=0.19$ ), increased non-HDL cholesterol $\left(p\right.$ value $=1.78 \times 10^{-31}$, beta $\left.=0.19\right)$, increased total cholesterol to HDL ratio ( $p$ value $=5.27 \times 10^{-17}$, beta $=0.13$ ), and increased triglycerides $\left(p\right.$ value $=4.47 \times 10^{-6}$, beta $=$ $0.03)$. LDL $L_{\mathrm{PGS}}$ also associated with four blood biomarkers, mean corpuscular hemoglobin ( $p$ value $=5.68 \times 10^{-8}$, beta $=-0.02)$, total protein in blood ( $p$ value $=2.18 \times 10^{-6}$, beta $=0.02)$, total protein in serum $\left(p\right.$ value $=3.00 \times 10^{-6}$, beta $=0.02$ ), and mean corpuscular hemoglobin concentration $\left(p\right.$ value $=1.50 \times 10^{-5}$, beta $\left.=-0.02\right)$. LDLPGS $_{\text {in }}$ the African ancestry group associated with LDL cholesterol $\left(p\right.$ value $=5.71 \times 10^{-63}$, beta $\left.=0.24\right)$ and increased total cholesterol $\left(p\right.$ value $=1.63 \times 10^{-53}$, beta $\left.=0.21\right)($ Fig. $3 \mathrm{~d}$, Additional file 2: Table S9).

The LabWAS of $\mathrm{TG}_{\mathrm{PGS}}$ was associated with several metabolic measurements (Fig. 3e, Additional file 2: Table S10), including increased TG ( $p$ value $<2.23 \times 10^{-308}$, beta $=0.28)$, followed by decreased HDL ( $p$ value $=$ $4.83 \times 10^{-148}$, beta $\left.=-0.14\right)$, increased total cholesterol to HDL ratio ( $p$ value $=2.95 \times 10^{-28}$, beta $=0.02$ ), increased blood glucose ( $p$ value $=1.20 \times 10^{-22}$, beta $=$ $0.04)$, increased lipemic index $\left(p\right.$ value $=1.57 \times 10^{-18}$, beta $=0.01)$, increased total blood cholesterol $(p$ value $=$ $1.25 \times 10^{-14}$, beta $\left.=0.04\right)$, increased glycated hemoglobin ( $p$ value $=5.69 \times 10^{-9}$, beta $\left.=0.04\right)$, increased bedside glucose $\left(p\right.$ value $=2.99 \times 10^{-7}$, beta $\left.=0.04\right)$, and increased non-HDL cholesterol $\left(p\right.$ value $=1.18 \times 10^{-6}$, beta $\left.=0.08\right)$.
Additionally, $\mathrm{TG}_{\mathrm{PGS}}$ showed associations with seven immune labs, white blood cells ( $p$ value $=3.90 \times 10^{-30}$, beta $=0.04)$, immature granulocytes $(p$ value $=1.99 \times$ $10^{-14}$, beta $\left.=0.03\right)$, absolute lymphocytes $(p$ value $=$ $2.01 \times 10^{-11}$, beta $=0.03$ ), monocyte to leukocyte ratio $\left(p\right.$ value $=5.21 \times 10^{-10}$, beta $\left.=-0.03\right)$, absolute neutrophils $\left(p\right.$ value $=1.87 \times 10^{-9}$, beta $\left.=0.03\right)$, complement C4 $\left(p\right.$ value $=1.03 \times 10^{-8}$, beta $\left.=0.09\right)$, and monocyte count $\left(p\right.$ value $=6.76 \times 10^{-8}$, beta $\left.=-0.03\right)$. Several blood associations also emerged with $\mathrm{TG}_{\mathrm{PGS}}$, including carbon dioxide ( $p$ value $=2.57 \times 10^{-24}$, beta $\left.=-0.04\right)$, total protein in blood $\left(p\right.$ value $=4.25 \times 10^{-16}$, beta $\left.=0.03\right)$, mean corpuscular volume $\left(p\right.$ value $=9.16 \times 10^{-13}$, beta $=-0.03$ ), mean corpuscular hemoglobin $\left(p\right.$ value $=9.75 \times 10^{-8}$, beta $=$ $-0.02)$, anion gap ( $p$ value $=2.03 \times 10^{-17}$, beta $\left.=0.03\right)$, total protein in serum $\left(p\right.$ value $=2.61 \times 10^{-16}$, beta $\left.=0.04\right)$, and calcitriol $\left(p\right.$ value $=1.07 \times 10^{-10}$, beta $\left.=-0.05\right)$. Lastly, $\mathrm{TG}_{\mathrm{PGS}}$ associated with albumin to creatinine ratio $(p$ value $=9.13 \times 10^{-8}$, beta $\left.=0.10\right)$, urate $\left(p\right.$ value $=6.58 \times 10^{-9}$, beta $=0.06)$, urinary $\mathrm{pH}\left(7.66 \times 10^{-7}\right.$, beta $\left.=-0.02\right)$, and urinary albumin concentration $\left(p\right.$ value $=2.99 \times 10^{-5}$, beta $=$ $0.06)$. In the African ancestry group, $\mathrm{TG}_{\mathrm{PGS}}$ showed significant associations with increased triglycerides ( $p$ value $=1.66 \times 10^{-53}$, beta $\left.=0.19\right)$, decreased HDL cholesterol ( $p$ value $=6.08 \times 10^{-11}$, beta $\left.=-0.08\right)$, and increased glucose $\left(p\right.$ value $=2.33 \times 10^{-5}$, beta $\left.=0.04\right)$ (Fig. 3f, Additional file 2: Table S11).

\section{LabWAS of a polygenic score for coronary artery disease}

We next sought to recapitulate the risk biomarker profile for CAD through a LabWAS of a CAD PGS. The CAD $_{\text {PGS }}$ reproduced associations, in the direction of risk, with canonical risk factors for CAD (Fig. 4a, Additional file 2: Table S12) in the European ancestry population, including decreased HDL ( $p$ value $=6.20 \times 10^{-39}$, beta $\left.=-0.07\right)$, increased TG $\left(p\right.$ value $=3.98 \times 10^{-25}$, beta $\left.=0.06\right)$, increased blood glucose $\left(p\right.$ value $=1.18 \times 10^{-21}$, beta $\left.=0.04\right)$ and glycated hemoglobin $\left(p\right.$ value $=2.36 \times 10^{-12}$, beta $\left.=0.05\right)$, and bedside glucose $\left(p\right.$ value $=1.10 \times 10^{-6}$, beta $=0.03$ ) The $\mathrm{CAD}_{\mathrm{PGS}}$ also associated with other known biomarkers of cardiovascular health such as increased troponin-I $\left(p\right.$ value $=7.20 \times 10^{-9}$, beta $\left.=0.04\right)$ and brain natriuretic peptide $\left(p\right.$ value $=2.12 \times 10^{-7}$, beta $\left.=0.05\right)$. $\mathrm{CAD}_{\mathrm{PGS}}$ associated with six blood composition markers, red blood cell distribution width $\left(p\right.$ value $=1.60 \times 10^{-11}$, beta $=0.03)$, mean corpuscular hemoglobin $(p$ value $=$ $6.73 \times 10^{-10}$, beta $\left.=-0.02\right)$, mean corpuscular volume $(p$ value $=1.17 \times 10^{-9}$, beta $\left.=-0.02\right)$, carbon dioxide ( $p$ value $=$ $3.36 \times 10^{-9}$, beta $\left.=-0.02\right)$, red blood cell sedimentation rate $\left(p\right.$ value $=2.10 \times 10^{-7}$, beta $\left.=0.05\right)$, and international normalized rate $\left(p\right.$ value $=1.96 \times 10^{-5}$, beta $\left.=0.03\right)$. Finally, $\mathrm{CAD}_{\mathrm{PGS}}$ associated with white blood cell count $(p$ value $=$ $8.75 \times 10^{-11}$, beta $\left.=0.02\right)$, creatinine $\left(p\right.$ value $=2.13 \times 10^{-6}$, 


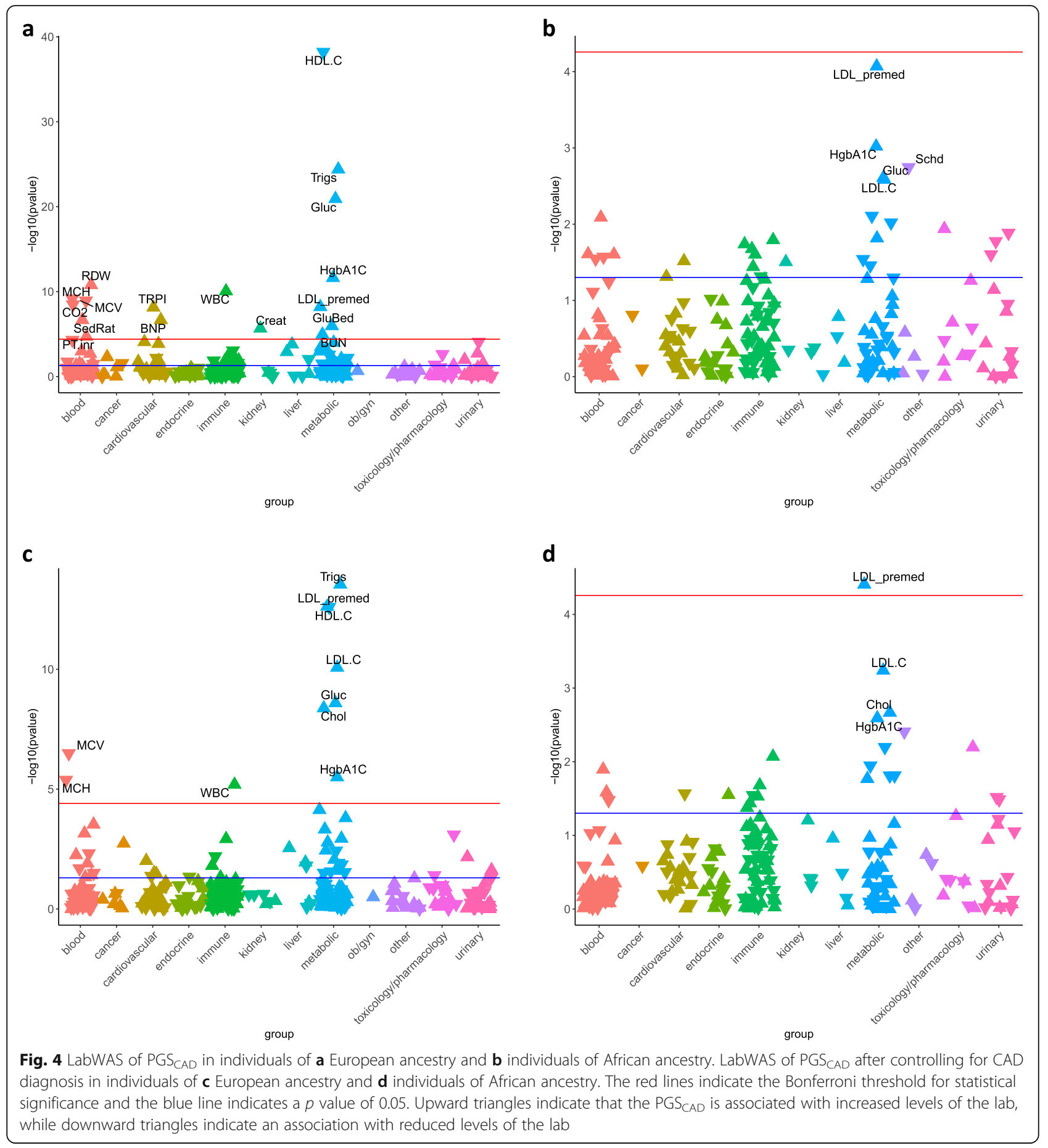

beta $=0.02)$, and blood urea nitrogen $\left(p\right.$ value $=1.09 \times 10^{-5}$, beta $=0.02$ ).

Notably, the $\mathrm{CAD}_{\mathrm{PGS}}$ was not initially associated with LDL values $(p$ value $=0.13$, beta $=0.008)$. The lack of association, however, was attributable to lipid altering medication use and a significant association between the CAD $_{\text {PGS }}$ and LDL levels was detected when we restricted to pre-medication values $\left(p=6.19 \times 10^{-9}\right.$, beta $\left.=0.04\right)$.
To determine which biomarkers were explained by the clinical presence of $\mathrm{CAD}$ as opposed to the genetic risk for CAD, we adjusted the LabWAS of $C_{A D} D_{\text {PGS }}$ for the coronary atherosclerosis phecode (411) (Fig. 4c, Additional file 2: Table S13). Four canonical biomarkers of CAD risk remained associated with $C_{\text {PGS }}$ including TG $\left(p\right.$ value $=2.88 \times 10^{-14}$, beta $\left.=0.05\right)$, pre-medication LDL $\left(p\right.$ value $=2.40 \times 10^{-13}$ beta $\left.=0.05\right)$, HDL $(p$ value $=$ 
$2.55 \times 10^{-13}$, beta $\left.=-0.04\right), \quad$ LDL-C $\quad(p$ value $=8.48 \times$ $10^{-11}$, beta $\left.=0.04\right)$, blood glucose $\left(p\right.$ value $=2.55 \times 10^{-9}$, beta $=0.02)$, total cholesterol $\left(p\right.$ value $=4.16 \times 10^{-9}$, beta $=0.03)$, and glycated hemoglobin $(p$ value $=3.16 \times$ $10^{-6}$, beta $\left.=0.03\right)$. The $\mathrm{CAD}_{\mathrm{PGS}}$ also remained associated with one immune marker, white blood cell count ( $p$ value $=6.44 \times 10^{-6}$, beta $\left.=0.02\right)$, and two other blood biomarkers, mean corpuscular volume $(p$ value $=3.23 \times$ $10^{-7}$, beta $\left.=-0.02\right)$ and mean corpuscular hemoglobin $\left(p\right.$ value $=4.18 \times 10^{-6}$, beta $\left.=-0.02\right)$.

None of the associations in the initial LabWAS of $\mathrm{CAD}_{\mathrm{PGS}}$ among African ancestry individuals reached phenome-wide significance; however, three of the top four associations were canonical CAD risk factors including increased glycated hemoglobin A1c ( $p$ value $=$ $9.56 \times 10^{-4}$, beta $\left.=0.04\right)$, increased glucose $(p$ value $=$ 0.002 , beta $=0.03)$, and increased LDL cholesterol $(p$ value $=0.003$, beta $=0.04) \quad($ Fig. $4 \mathrm{~b}$, Additional file 2: Table S14). When the LDL levels were restricted to pre-medication values, the top association with CAD $_{\text {PGS }}$ was pre-medication LDL ( $p$ value $=8.50 \times$ $10^{-5}$, beta $=0.06$ ); however, this association did not pass multiple testing correction. After controlling the analysis for CAD diagnosis, the association between $\mathrm{CAD}_{\mathrm{PGS}}$ and pre-medication LDL surpassed the Bonferroni correction for phenome-wide significance $\left(p \quad\right.$ value $=3.92 \times 10^{-5}, \quad$ beta $\left.=0.06\right) \quad($ Fig. $4 d, \quad$ Additional file 2: Table S15).

Lastly, we ran a LabWAS of CAD diagnosis (i.e., using CAD cases/control status (Additional file 1) as the predictor variable) after adjusting for sex and median age across the EHR, which revealed the medical comorbidity pattern of CAD. CAD diagnosis was significantly associated with 136 out of 734 labs in our sample (Additional file 3: Fig. S7, Additional file 2: Table S15), including 34 immune, 32 blood, 24 metabolic, 17 cardiovascular, 8 urinary, 5 toxicology/pharmacology, 4 endocrine, 3 kidney, 3 liver, 1 cancer, and 5 other markers.

\section{Replication in Mass General Brigham Biobank}

In the MGBB, there were 21,499 individuals of European descent with genetic data available with recorded lab data. Slightly more than half of the sample was female (51.5\%) and the average age was 56.1 years. The MGBB patients contained 1094 CAD cases and 20,405 CAD controls.

In MGBB, the HDL $\mathrm{PGS}_{\mathrm{PS}}$ most strongly associated with HDL cholesterol ( $p$ value $<2.23 \times 10^{-308}$, beta $=0.33$ ), followed by decreased triglycerides $\left(p\right.$ value $=2.77 \times 10^{-109}$, beta $=-0.17)$, increased total cholesterol $(p$ value $=4.96 \times$ $10^{-31}$, beta $=0.09$ ), and decreased very low-density lipoprotein $\left(p\right.$ value $=2.62 \times 10^{-29}$, beta $\left.=-0.14\right)$. HDL $_{\mathrm{PGS}}$ also associated with decreased values of glucose $(p$ value $=$ $3.33 \times 10^{-27}$, beta $\left.=-0.07\right)$, hemoglobin A1c $(p$ value $=$
$3.64 \times 10^{-18}$, beta $\left.=-0.07\right)$, and mean glucose value $(p$ value $=4.10 \times 10^{-17}$, beta $\left.=-0.07\right)$. Additional associations with $\mathrm{HDL}_{\mathrm{PGS}}$ included cardiac relative risk ( $p$ value $=$ $5.72 \times 10^{-17}$, beta $\left.=-0.20\right)$, alanine aminotransferase $(p$ value $=2.45 \times 10^{-10}$, beta $\left.=-0.04\right)$, white blood cell count ( $p$ value $=1.03 \times 10^{-9}$, beta $\left.=-0.04\right)$, mean corpuscular volume $\left(p\right.$ value $=6.51 \times 10^{-8}$, beta $\left.=0.03\right)$, non-HDL cholesterol $\left(p\right.$ value $=1.41 \times 10^{-7}$, beta $\left.=-0.06\right)$, red blood cell distribution width $\left(p\right.$ value $=2.60 \times 10^{-7}$, beta $\left.=-0.03\right)$, neutrophils $\left(p\right.$ value $=2.95 \times 10^{-7}$, beta $\left.=-0.03\right)$, urate $(p$ value $=1.79 \times 10^{-6}$, beta $\left.=-0.05\right)$, and alkaline phosphatase ( $p$ value $=2.01 \times 10^{-6}$, beta $\left.=-0.03\right) \quad$ (Additional file 3 : Fig. 8a, Additional file 2: Table S17).

The LDL $_{\mathrm{PGS}}$ associated with four metabolic labs including LDL-C ( $p$ value $=1.78 \times 10^{-158}$, beta $\left.=0.24\right)$, total cholesterol $\left(p\right.$ value $=2.37 \times 10^{-158}$, beta $\left.=0.20\right)$, calculated LDL cholesterol $\left(p\right.$ value $=1.28 \times 10^{-81}$, beta $=$ 0.23 ), and non-HDL cholesterol $\left(p\right.$ value $=2.90 \times 10^{-68}$, beta $=0.19)$. The LDL $L_{\text {PGS }}$ also associated with complement C4 $\left(p\right.$ value $=1.85 \times 10^{-5}$, beta $\left.=0.09\right)$, red blood cell sedimentation rate $\left(p\right.$ value $=2.60 \times 10^{-5}$, beta $=$ $0.04)$, and increased cardiac relative risk ( $p$ value $=$ $3.80 \times 10^{-5}$, beta $=0.10$ ) (Additional file 3: Fig. $8 \mathrm{~b}$, Additional file 2: Table S18).

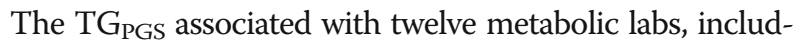
ing increased measured triglycerides ( $p$ value $<2.23 \times$ $10^{-308}$, beta $=0.32$ ), followed by increased very low-density lipoprotein $\left(p\right.$ value $=8.90 \times 10^{-129}$, beta $\left.=0.30\right)$, decreased HDL $\left(p\right.$ value $=1.33 \times 10^{-123}$, beta $\left.=-0.17\right)$, increased nonHDL cholesterol $\left(p\right.$ value $=8.70 \times 10^{-28}$, beta $\left.=0.12\right)$, increased glucose $\left(p\right.$ value $=4.56 \times 10^{-14}$, beta $\left.=0.05\right)$, average glucose $\left(p\right.$ value $=4.16 \times 10^{-10}$, beta $\left.=0.05\right)$, total cholesterol $\left(p\right.$ value $=1.58 \times 10^{-9}$, beta $\left.=0.05\right)$, anion gap $(p$ value $=1.52 \times 10^{-7}$, beta $\left.=0.03\right)$, total protein $(p$ value $=$ $4.63 \times 10^{-7}$, beta $\left.=0.03\right)$, globulin in serum $(p$ value $=$ $8.80 \times 10^{-6}$, beta $\left.=0.03\right)$, aspartate aminotransferase $(p$ value $=1.26 \times 10^{-5}$, beta $\left.=0.03\right)$, and sodium $(p$ value $=$ $1.27 \times 10^{-5}$, beta $\left.=-0.03\right) . \mathrm{TG}_{\mathrm{PGS}}$ also associated with seven immune labs, white blood cell count $\left(p\right.$ value $=3.89 \times 10^{-17}$, beta $=0.05)$, lymphocytes $\left(p\right.$ value $=7.86 \times 10^{-11}$, beta $=$ $0.04)$, complement $C 4$ ( $p$ value $=1.58 \times 10^{-9}$, beta $\left.=0.13\right)$, automated lymphocyte count $\left(p\right.$ value $=2.14 \times 10^{-9}$, beta $=$ $0.09)$, neutrophils $\left(p\right.$ value $=3.09 \times 10^{-7}$, beta $\left.=0.05\right)$, automated neutrophil count $\left(p\right.$ value $=5.13 \times 10^{-7}$, beta $\left.=0.03\right)$, and monocytes $\left(p\right.$ value $=3.38 \times 10^{-6}$, beta $\left.=0.05\right)$. Ten additional labs significantly associated with $\mathrm{TG}_{\mathrm{PGS}}$, including increased cardiac relative risk $\left(p\right.$ value $=5.49 \times 10^{-15}$, beta $=0.19)$, mean corpuscular volume $(p$ value $=3.02 \times$ $10^{-14}$, beta $\left.=-0.05\right)$, glycated hemoglobin A1c $(p$ value $=$ $5.00 \times 10^{-11}$, beta $\left.=0.05\right)$, urinary $\mathrm{pH} \quad(p$ value $=9.58 \times$ $10^{-10}$, beta $\left.=-0.04\right)$, red blood cell sedimentation rate $\left(p\right.$ value $=2.22 \times 10^{-8}$, beta $\left.=0.05\right)$, alanine aminotransferase $\quad\left(p \quad\right.$ value $=3.88 \times 10^{-8}, \quad$ beta $\left.=0.04\right), \quad$ alkaline phosphatase $\left(p\right.$ value $=3.17 \times 10^{-7}$, beta $\left.=0.03\right)$, blood 
carbon dioxide $\left(p\right.$ value $=5.63 \times 10^{-7}$, beta $\left.=-0.03\right)$, mean corpuscular hemoglobin $\left(p\right.$ value $=1.49 \times 10^{-6}$, beta $=-0.03)$, and urate $\left(p\right.$ value $=1.62 \times 10^{-6}$, beta $=$ 0.05) (Additional file 3: Fig. 8c, Additional file 2: Table S19).

Finally, the $\mathrm{CAD}_{\mathrm{PGS}}$ associated with several known $\mathrm{CAD}$ risk factors, including decreased HDL-C $(p$ value $=$ $1.56 \times 10^{-21}$, beta $\left.=-0.07\right)$, increased glucose $(p$ value $=$ $9.91 \times 10^{-15}$, beta $\left.=0.05\right)$, increased glycated hemoglobin A1c $\left(p\right.$ value $=4.44 \times 10^{-14}$, beta $\left.=0.06\right)$, mean glucose $(p$ value $=1.75 \times 10^{-12}$, beta $\left.=0.06\right)$, and increased triglycerides $\left(p\right.$ value $=2.09 \times 10^{-12}$, beta $\left.=0.05\right)$. The $\mathrm{CAD}_{\mathrm{PGS}}$ also associated with increased red blood cell distribution width $\left(p\right.$ value $=2.42 \times 10^{-14}$, beta $\left.=0.05\right)$, increased red blood cell sedimentation rate $\left(p\right.$ value $=4.11 \times 10^{-9}$, beta $=0.05)$, increased alanine aminotransferase $(p$ value $=2.59 \times 10^{-8}$, beta $\left.=0.04\right)$, decreased hemoglobin ( $p$ value $=1.45 \times 10^{-6}$, beta $\left.=-0.03\right)$, increased alkaline phosphatase $\left(p\right.$ value $=2.26 \times 10^{-6}$, beta $\left.=0.03\right)$, increased white blood cell count $\left(p\right.$ value $=6.77 \times 10^{-6}$, beta $=0.03)$, decreased albumin $\left(p\right.$ value $=1.06 \times 10^{-5}$, beta $=-0.03)$, increased globulin $\left(p\right.$ value $=1.29 \times 10^{-5}$, beta $=0.03)$, decreased iron $\left(p\right.$ value $=3.36 \times 10^{-5}$, beta $=$ $-0.04)$, and decreased hematocrit ( $p$ value $=3.77 \times 10^{-5}$, beta $=-0.03$ ) (Additional file 3: Fig. 9a, Additional file 2: Table S20).

After adjusting for CAD diagnosis, $\mathrm{CAD}_{\mathrm{PGS}}$ remained associated with several heart disease risk factors including decreased HDL-C ( $p$ value $=8.23 \times 10^{-16}$, beta $\left.=-0.06\right)$, increased glucose $\left(p\right.$ value $=6.80 \times 10^{-11}$, beta $\left.=0.04\right)$, increased hemoglobin A1c ( $p$ value $=2.08 \times 10^{-10}$, beta $=$ $0.05)$, increased mean glucose $\left(p\right.$ value $=2.29 \times 10^{-9}$, beta $=$ 0.05 ), and increased triglycerides ( $p$ value $=3.48 \times 10^{-9}$, beta $=0.05$ ). Additionally, associations with red blood cell distribution width $\left(p\right.$ value $=1.40 \times 10^{-12}$, beta $\left.=0.04\right)$, alanine aminotransferase $\left(p\right.$ value $=4.01 \times 10^{-8}$, beta $=0.04$ ), red blood cell sedimentation rate $\left(p\right.$ value $=5.50 \times 10^{-8}$, beta $=0.05)$, alkaline phosphatase $\left(p\right.$ value $=5.44 \times 10^{-6}$, beta $=0.03)$, serum globulin $\left(p\right.$ value $=4.51 \times 10^{-5}$, beta $=$ 0.03 ), and white blood cell count ( $p$ value $=4.67 \times 10^{-5}$, beta $=0.03$ ) remained (Additional file 3: Fig. 9b, Additional file 2: Table S21). In MGBB, the $\mathrm{CAD}_{\mathrm{PGS}}$ was not associated with levels of LDL-C ( $p$ value $=0.06$, beta $=-0.03$ ), and we were unable to investigate the effects of cholesterol lowering medications on the association.

\section{Discussion}

The results of our study add to a growing body of evidence indicating that lab values from EHRs with linked genetic data can be mined at scale to identify biomarkers for complex disease [1-5]. Our proof-of-principle analyses focused on lipids and CAD in 94,747 genotyped BioVU patients and revealed that EHR lipid values cleaned using our QualityLab pipeline were genetically comparable to those measured in samples ascertained for research. Here, we describe two proof concept studies that demonstrate the power of our proposed discovery paradigm. First, we show that PGS for lipids (HDL, LDL, and triglycerides) associate robustly to their referent lipid across ancestries (Fig. 3). Moreover, the $\mathrm{CAD}_{\mathrm{PGS}}$ recapitulated associations with known biomarkers in individuals of European ancestry in two biobanks. Unlike CAD, many complex diseases do not yet have bona fide biomarkers, but do have well-powered GWAS that can be used to mine large biobanks and identify quantitative labs which may be correlated, even weakly, with genetic risk for disease. Importantly, the association between $C_{A D} D_{\mathrm{PGS}}$ and canonical risk factors was significant even among those who did not have a CAD diagnosis. In analyses in Mass General Brigham Biobank, several of the associations with $\mathrm{CAD}_{\mathrm{PGS}}$ replicated, helping to validate our approach to cleaning and analyzing EHR laboratory data. Interestingly, $\mathrm{CAD}_{\mathrm{PGS}}$ also associated with white blood cell count, an inflammatory marker that is not currently used to diagnose or monitor heart disease. This association remained after controlling for $\mathrm{CAD}$ diagnosis, indicating that $\mathrm{CAD}$ genetics could play a role in increasing inflammation. These results highlight the usefulness of our approach which takes advantage of the entire patient population regardless of disease status. This approach offers a potential path forward for the detection of novel biomarkers and for improved understanding of biomarker activity during the prodromal phase of disease. Furthermore, while disease PGS are not diagnostic, they may be useful in identifying pre-symptomatic individuals whose lab values should be monitored more closely.

Furthermore, we show that treatments (in this example, lipid-altering medications) can influence the detection of risk biomarkers at the genetic level. For example, we found that the genetic correlation between LDL measurements in BioVU and MVP increased considerably when we restricted to pre-medication LDL measurements and controlled for CAD or diabetes diagnosis. Additionally, the $\mathrm{CAD}_{\mathrm{PGS}}$ was strongly associated with pre-medication median LDL values, but was not associated with combined pre- and post-medication median LDL values. This finding also has important and complex implications for the clinical use of PGS recently discussed in the literature $[35,36]$. These results indicate that as preventative treatments for complex diseases are adopted (e.g., lipid-altering medications), the risk factors targeted by those treatments (e.g., lipids) are less likely to play a role in the development of subsequent disease (e.g., CAD) in current and future treated populations. Thus, today's PGS will no longer identify at-risk individuals in future generations who are routinely treated for risk factors which are only now being discovered. 
Moreover, cases ascertained today for GWAS of diseases with available preventative treatments will be enriched for a different set of genetic (and environmental) risk factors because those individuals with risk factors that can be treated are less likely to develop the disease. PGS, while incredibly valuable, provide only a snapshot of the human genetic profile of complex disease and thus are highly susceptible to these types of cohort effects in addition to other known sources of technical and experimental artifacts $[37,38]$.

Though the results and approach presented provide an exciting path forward for genetic analysis of EHR-lab data, important limitations should be acknowledged. First, our analyses yielded more associations in patients of European ancestry compared to patients of African ancestry. This is likely to due to decreased power from both the discovery GWASs and the target sample. BioVU has considerably fewer patients of African ancestry than European ancestry, impacting our statistical power to find associations. The polygenic scores of lipids, which were trained on trans-ancestry GWAS summary statistics including individuals of African descent, strongly associated with the referent lipid in the African ancestry sample with effect estimates similar to those found in the European sample. However, the CAD polygenic score, which was trained on a trans-ancestry GWAS that did not include African ancestry samples, yielded far fewer significant associations. These results highlight the critical importance of diversity in GWAS as the downstream applications of such studies are dramatically impacted by representation. As the number of ancestrally diverse GWAS increase, so too will our ability to identify novel biomarkers in different ancestral groups, and the QualityLab pipeline is poised to deliver on these analyses. The QualityLab pipeline could also have more immediate clinical impact for diverse populations. Genetic ancestry, race, sex, age, and ethnicity strongly influence the distribution of lab tests results in healthy people [39], but many current reference ranges were developed using White middle-aged men and are applied to patients irrespective of these differences. This could result in under- or over-diagnosis in some patient groups, and developing lab reference ranges appropriate for diverse demographics is low-hanging fruit for precision medicine. The QualityLab pipeline provides summary metrics based on demographic features which allows the user to evaluate lab distributions across populations, sexes, and ages.

Second, polygenic scores are based on GWAS summary statistics that are typically unadjusted for phenotypic comorbidities. While this approach is optimal in GWAS for many reasons, it introduces the possibility of "phenotypic hitchhiking" in which a comorbid trait is unintentionally selected during the ascertainment of the index trait. Thus, two heritable phenotypes that might share common environmental risk factors but no genetic risk factors can subsequently appear correlated in PGS analysis, even in independent samples. We therefore emphasize that this genetic approach is still fundamentally correlational.

Third, high-throughput analysis of 939 lab traits in our LabWAS required us to prioritize statistical model performance over coefficient interpretability. In our primary analysis, we transformed lab values to fit the normal distribution to improve the performance of the linear regression models [21]. We applied the rank-based inverse normal quantile transformation to all labs, which ensured trait normality by replacing the value of each observation with its quantile from the standard normal distribution. The inverse normal quantile transformation thus preserved the rank ordering of observations, but not the values themselves, and model coefficients therefore are uninterpretable on the original scale. For example, based on our LabWAS results, we are unable to report the change in LDL levels in $\mathrm{mg} / \mathrm{dL}$ per SD increase in the $\mathrm{CAD}_{\mathrm{PGS}}$. Multiple testing correction was another statistical challenge inherent to the high-throughput analysis of lab traits. We used the Bonferroni threshold for statistical significance, but this threshold is likely to be overly strict because it ignores the correlation between lab tests.

\section{Conclusions}

Here, we propose that PGS for complex disease can be used to discover genetically related biomarkers of disease by mining quantitative physiological measurements collected during routine clinical testing, but caution that mindful interpretation of correlational results is paramount to progress. We demonstrate the robustness of this discovery paradigm in a proof of principal analysis focused on CAD. As EHR resources grow in size, standardized quality control and analysis pipelines will be necessary to compare results across samples. QualityLab and LabWAS provide a starting point for consistent analysis of lab results stored in various EHR systems. Furthermore, we demonstrated that EHR-derived lipids are similar to measurements ascertained in traditional cohort studies, providing additional rationale for analyses of EHR labs [40]. QualityLab and LabWAS are scalable programs that can be used to confirm clinical paradigms and discover new genetic and environmental relationships between biomarkers and complex traits. We propose that future studies will leverage this discovery paradigm for analysis of rare or understudied complex traits with no known biomarker associations (e.g., psychiatric disorders). 


\section{Supplementary Information}

The online version contains supplementary material available at https://doi. org/10.1186/s13073-020-00820-8

Additional file 1. Contains supplementary methods information on genotyping and quality control, definition of coronary artery disease and lipids lowering mediation, and polygenic scoring methods.

Additional file 2: Contains all supplementary tables. Table S1. Descriptive statistics generated by the QualityLab pipeline. Table S2. Lipid-altering medications abstracted from free text in clinical notes. Table S3. Characteristics of patients with clean lab and genotyping data. Table S4. Estimates of SNP-based heritability and sample sizes for all labs passing QualityLab using REML. Table S5. Estimates of SNP-based heritability for blood lipid levels across study populations and estimation methods. Table S6. LabWAS of HDLpgs in the European sample in BioVU. Table S7. LabWAS of HDLpGS in the African sample in BioVU. Table S8. LabWAS of LDLpGS in the European sample in BioVU. Table S9. LabWAS of LDLPGS in the African sample in BioVU. Table S10. LabWAS of TGPGS in the European sample in BioVU. Table S11. LabWAS of TG PGS $_{\text {in }}$ the African sample in BioVU. Table S12. LabWAS of CADPGS in the European sample in BioVU. Table S13. LabWAS of CADPGS in the European sample in BioVU controlling for CAD diagnosis. Table S14. LabWAS of CADPGS in the African sample in BioVU. Table S15. LabWAS of CADPGS in the African sample in BioVU controlling for CAD diagnosis. Table S16. LabWAS of CAD diagnosis in the European sample in BioVU. Table S17. LabWAS of HDLPGS in the European sample in MGB. Table S18. LabWAS of LDLPGS $_{\text {in the European sample in MGB. Table S19. LabWAS of TG }}$ in the European sample in MGB. Table S20. LabWAS of CADPGS in the European sample in MGB. Table S21. LabWAS of CADPGS in the European sample in MGB controlling for CAD diagnosis. Table S22. The GWAS Catalog study accession numbers for summary statistics from GWAS of each lab trait.

Additional file 3: Contains all supplementary figures. Figure S1. QualityLab data visualizations. Figure S2. Manhattan and QQ plots from GWAS of BioVU lipids. Figure S3. Predictive abilities of different polygenic scoring methods. Figure S4. Lipids levels by genetic ancestry. Figure S5. Histogram of BioVU lab heritability estimates. Figure S6. LDL genetic correlation sensitivity analyses. Figure S7. LabWAS Manhattan of CAD diagnosis. Figure S8. LabWAS of Lipids PGS in MGBB. Figure $\mathbf{S 9 .}$ LabWAS of CAD PGS in MGBB.

\section{Abbreviations}

Lab: Laboratory; EHR: Electronic health record; PGS: Polygenic score; LabWAS: Lab-wide association scan; PheWAS: Phenome-wide association scan; VUMC: Vanderbilt University Medical Center; HDL: High-density lipoprotein; LDL: Low-density lipoprotein; TG: Triglycerides; CAD: Coronary artery disease; ICD: International Classification of Disease; CPT: Current Procedural Terminology; HRC: Haplotype Reference Consortium; GWAS: Genome-wide association scan; INT: Inverse normal quantile

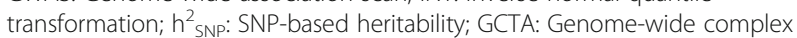
trait analysis; REML: Restricted maximum likelihood; GLGC: Global Lipids Genetics Consortium; PRS-CS: Polygenic Risk Score - Continuous Shrinkage

\section{Acknowledgements}

The authors thank the Vanderbilt University Medical Center Biobank and Mass General Brigham Biobank for providing genomic and health information data.

\section{Authors' contributions}

JKD, JMS, GC, and LKD conceived and designed the study. All authors contributed to the acquisition, analysis, or interpretation of data. JKD, JMS, and LKD drafted the manuscript. All authors contributed to revision of the manuscript. All authors read and approved the final manuscript.

\section{Funding}

JKD is supported by the Canadian Institutes of Health Research (award MFE142936). JMS is supported by NIH/NIGMS training grant 5T32GM080178-12. JDM is supported by AHA grant 16FTF30130005 and NIH GM130791-01. GC is supported by NIH UL1TR000427 and 1U24CA242637-01. LKD is supported by NIH 1R01MH118233-01, 5U54MD010722-04, 5R01MH113362-03, and
1R56MH120736-01. JWS is supported by NIH R01 MH118233. DMR is supported by 5R01MH113362, R01MH11629, R01MH120736, and R01MH1 18233. This project was conducted in part using the resources of the Advanced Computing Center for Research and Education at Vanderbilt University, Nashville, TN. The datasets used for this project were obtained from Vanderbilt University Medical Center's Synthetic Derivative, which is supported by numerous sources: institutional funding, private agencies, and federal grants. These include the NIH funded Shared Instrumentation Grant S10RR025141 and CTSA grants UL1TR002243, UL1TR000445, and UL1RR024975. The project described was supported by the National Center for Research Resources, Grant UL1 RR024975-01, and is now at the National Center for Advancing Translational Sciences, Grant 2 UL1 TR000445-06. The content is solely the responsibility of the authors and does not necessarily represent the official views of the $\mathrm{NIH}$.

\section{Availability of data and materials}

The data that support the findings of this study are available from Vanderbilt University Medical Center but restrictions apply to the availability of these data, which were used under license for the current study, and so are not publicly available. Data are however available from the authors upon reasonable request and with permission of Vanderbilt University Medical Center.

GWAS data generated are available on the GWAS Catalog (accession numbers GCST90012603 - GCST90012784 are listed in Additional file 2: Table S22) [41] and on https://www.dropbox.com/sh/w1pbe0jq1bjkpc5/ AAAUldtBgUybE6iHraE8jvp8a?dl=0.

Code for QualityLab and LabWAS software used to generate the results presented in this paper can be found here (https://bitbucket.org/straubp_vandy/quality_ labs/) [42] and here (https://bitbucket.org/juliasealock/labwas/) [43].

LabWAS plots can be viewed interactively here: https://dennislab.ca/labwasin-electronic-health-records/

\section{Ethics approval and consent to participate}

BioVU Consent form is provided to patients in the outpatient clinic environments at VUMC. The consent states policies on data sharing and privacy and, upon consent, makes any blood leftover from clinical care eligible for BioVU banking. The VUMC Institutional Review Board oversees BioVU and approved this project. All data included in this study was deidentified and unlinked to any identifying information. This study was reviewed by the Vanderbilt University Medical Center IRB (IRB\#172020) and designated as non-human subjects research. The research was conducted in accordance with the principles of the Declaration of Helsinki.

\section{Consent for publication}

Not applicable

\section{Competing interests}

JWS is an unpaid member of the Bipolar/Depression Research Community Advisory Panel of 23andMe, is a member of the Leon Levy Foundation Neuroscience Advisory Board, and received an honorarium for an internal seminar at Biogen, Inc. He is PI of a collaborative study of the genetics of depression and bipolar disorder sponsored by 23andMe for which 23andMe provides analysis time as in-kind support but no payments. The remaining authors declare that they have no competing interests.

\section{Author details}

${ }^{1}$ Division of Genetic Medicine, Department of Medicine, Vanderbilt University Medical Center, Nashville, TN 37232, USA. 'ªnderbilt Genetics Institute, Vanderbilt University Medical Center, Nashville, TN 37232, USA. ${ }^{3}$ Department of Medical Genetics, University of British Columbia, Vancouver, BC V5Z 4H4, Canada. ${ }^{4}$ Psychiatric \& Neurodevelopmental Genetics Unit, Center for Genomic Medicine, Massachusetts General Hospital, Boston, MA 02114, USA. ${ }^{5}$ Department of Psychiatry, Harvard Medical School, Boston, MA 02115, USA. ${ }^{6}$ Stanley Center for Psychiatric Research, Broad Institute of Harvard and MIT, Cambridge, MA 02142, USA. 'Department of Microbiology, Immunology, and Physiology, Meharry Medical College, Nashville, TN 37232, USA. ${ }^{8}$ Analytic and Translational Genetics Unit, Center for Genomic Medicine, Massachusetts General Hospital, Boston, MA 02114, USA. ${ }^{9}$ Department of Molecular Physiology and Biophysics, Vanderbilt University Medical Center, Nashville, TN 37232, USA. ${ }^{10}$ Department of Psychiatry and Behavioral Sciences, Vanderbilt University Medical Center, Nashville, TN 37232, USA. ${ }^{11}$ Departments of Medicine and Biomedical Informatics, Vanderbilt University Medical Center, 
Nashville, TN 37232, USA. ${ }^{12}$ Department of Biostatistics and Medical Informatics, University of Wisconsin-Madison, Madison, WI 53706, USA. ${ }^{13}$ Division of Genetic Medicine, Department of Medicine, Vanderbilt Genetics Institute, Vanderbilt University, 511-A Light Hall, 2215 Garland Ave, Nashville, TN 37232, USA.

\section{Received: 25 February 2020 Accepted: 8 December 2020}

Published online: 13 January 2021

\section{References}

1. Shameer K, Denny JC, Ding K, Jouni H, Crosslin DR, de Andrade M, et al. A genome- and phenome-wide association study to identify genetic variants influencing platelet count and volume and their pleiotropic effects. Hum Genet. 2014;133:95-109.

2. Hoffmann TJ, Theusch E, Haldar T, Ranatunga DK, Jorgenson E, Medina MW et al. A large electronic-health-record-based genome-wide study of serum lipids. Nat Genet. 2018. https://doi.org/10.1038/s41588-018-0064-5.

3. Verma A, Lucas A, Verma SS, Zhang Y, Josyula N, Khan A, et al. PheWAS and beyond: the landscape of associations with medical diagnoses and clinical measures across 38,662 individuals from Geisinger. Am J Hum Genet. 2018 https://doi.org/10.1016/j.ajhg.2018.02.017.

4. Klarin D, Damrauer SM, Cho K, Sun YV, Teslovich TM, Honerlaw J, et al. Genetics of blood lipids among $\sim 300,000$ multi-ethnic participants of the million veteran program. Nat Genet. 2018;50:1514-23.

5. Verma A, Leader JB, Verma SS, Frase A, Wallace J, Dudek S, et al. Integrating clinical laboratory measures and ICD-9 code diagnoses in phenome-wide association studies. Pac Symp Biocomput. 2016. https://doi.org/10.1142/ 9789814749411_0016.

6. Estiri H, Klann JG, Murphy SN. A clustering approach for detecting implausible observation values in electronic health records data. BMC Med Inform Decis Mak. 2019. https://doi.org/10.1186/s12911-019-0852-6.

7. Pivovarov R, Albers DJ, Sepulveda JL, Elhadad N. Identifying and mitigating biases in EHR laboratory tests. J Biomed Inform. 2014. https://doi.org/10. 1016/j.jbi.2014.03.016.

8. Perrotta PL, Karcher DS. Validating laboratory results in electronic health records: a college of American pathologists Q-probes study. Arch Pathol Lab Med. 2016. https://doi.org/10.5858/arpa.2015-0320-CP.

9. Denny JC, Bastarache L, Roden DM. Phenome-wide association studies as a tool to advance precision medicine. Annu Rev Genomics Hum Genet. 2016; 17:353-73.

10. Denny JC, Bastarache L, Ritchie MD, Carroll RJ, Zink R, Mosley JD, et al. Systematic comparison of phenome-wide association study of electronic medical record data and genome-wide association study data. Nat Biotechnol. 2013:31:1102-10.

11. Pendergrass SA, Brown-Gentry K, Dudek S, Frase A, Torstenson ES, Goodloe $R$, et al. Phenome-wide association study (PheWAS) for detection of pleiotropy within the Population Architecture using Genomics and Epidemiology (PAGE) network. Plos Genet. 2013;9. https://doi.org/10.1371/ journal.pgen.1003087.

12. Robinson JR, Denny JC, Roden DM, Van Driest SL. Genome-wide and phenome-wide approaches to understand variable drug actions in electronic health records. Clin Transl Sci. 2018;11:112-22.

13. Lucas AM, Palmiero NE, McGuigan J, Passero K, Zhou J, Orie D, et al. CLAR ITE facilitates the quality control and analysis process for EWAS of metabolic-related traits. Front Genet. 2019. https://doi.org/10.3389/fgene. 2019.01240

14. Roden DM, Pulley JM, Basford MA, Bernard GR, Clayton EW, Balser JR, Masys DR. Development of a large-scale de-identified DNA biobank to enable personalized medicine. Clin Pharmacol Ther. 2008. https:/doi.org/10.1038/ clpt.2008.89.

15. Purcell $S$, Neale B, Todd-Brown K, Thomas L, Ferreira MAR, Bender D, et al. PLINK: a tool set for whole-genome association and population-based linkage analyses. Am J Hum Genet. 2007;81:559-75.

16. Price AL, Patterson NJ, Plenge RM, Weinblatt ME, Shadick NA, Reich D. Principal components analysis corrects for stratification in genome-wide association studies. Nat Genet. 2006:38:904-9.

17. Patterson N, Price AL, Reich D. Population structure and eigenanalysis. Plos Genet. 2006:2:2074-93.

18. Das S, Forer L, Schönherr S, Sidore C, Locke AE, Kwong A, et al. Nextgeneration genotype imputation service and methods. Nat Genet. 2016:48: 1284-7.
19. Auton A, Abecasis GR, Altshuler DM, Durbin RM, Bentley DR, Chakravarti A, et al. A global reference for human genetic variation. Nature. 2015; 526:68-74.

20. Sofer T, Zheng X, Gogarten SM, Laurie CA, Grinde K, Shaffer JR, et al. A fully adjusted two-stage procedure for rank-normalization in genetic association studies. Genet Epidemiol. 2019. https://doi.org/10.1002/gepi.22188.

21. McCaw ZR, Lane JM, Saxena R, Redline S, Lin X. Operating characteristics of the rank-based inverse normal transformation for quantitative trait analysis in genome-wide association studies. Biometrics. 2019. https://doi.org/10. 1111/biom.13214.

22. Davis LK, Yu D, Keenan CL, Gamazon ER, Konkashbaev Al, Derks EM, et al. Partitioning the heritability of Tourette syndrome and obsessive compulsive disorder reveals differences in genetic architecture. Plos Genet. 2013. https://doi.org/10.1371/journal.pgen.1003864.

23. Yang J, Lee SH, Goddard ME, Visscher PM. GCTA: a tool for genome-wide complex trait analysis. Am J Hum Genet. 2011;88:76-82.

24. Zeng P, Zhao Y, Qian C, Zhang L, Zhang R, Gou J, et al. Statistical analysis for genome-wide association study. J Biomed Res. 2015. https://doi.org/10. 7555/JBR.29.20140007.

25. Jiang $L$, Zheng Z, Qi T, Kemper KE, Wray NR, Visscher PM, Yang J. A resource-efficient tool for mixed model association analysis of large-scale data. Nat Genet 2019;51:1749-55. https://doi.org/10.1038/s41588-019-0530-8.

26. Willer CJ, Schmidt EM, Sengupta S, Peloso GM, Gustafsson S, Kanoni S, et al. Discovery and refinement of loci associated with lipid levels. Nat Genet. 2013;45:1274-85.

27. Bulik-Sullivan B, Loh PR, Finucane HK, Ripke S, Yang J, Patterson N, et al. LD score regression distinguishes confounding from polygenicity in genomewide association studies. Nat Genet. 2015. https://doi.org/10.1038/ng.3211.

28. Evans LM, Tahmasbi R, Vrieze SI, Abecasis GR, Das S, Gazal S, et al. Comparison of methods that use whole genome data to estimate the heritability and genetic architecture of complex traits. Nat Genet. 2018. https://doi.org/10.1038/s41588-018-0108-x.

29. Bulik-Sullivan B, Finucane HK, Anttila V, Gusev A, Day FR, Loh PR, et al. An atlas of genetic correlations across human diseases and traits. Nat Genet. 2015;47:1236-41.

30. Ning Z, Pawitan $Y$, Shen $X$. High-definition likelihood inference of genetic correlations across human complex traits. Nat Genet. 2020. https://doi.org/ 10.1038/s41588-020-0653-y.

31. Ge T, Chen CY, Ni Y, Feng YCA, Smoller JW. Polygenic prediction via Bayesian regression and continuous shrinkage priors. Nat Commun. 2019;10: $1-10$

32. Nikpay M, Goel A, Won HH, Hall LM, Willenborg C, Kanoni S, et al. A comprehensive 1000 Genomes-based genome-wide association metaanalysis of coronary artery disease. Nat Genet. 2015;47:1121-30.

33. Choi SW, O'Reilly PF. PRSice-2: Polygenic Risk Score software for biobankscale data. Gigascience. 2019:8:1-6.

34. Karlson EW, Boutin NT, Hoffnagle AG, Allen NL. Building the partners healthcare biobank at partners personalized medicine: informed consent, return of research results, recruitment lessons and operational considerations. J Pers Med. 2016;6:1-11.

35. Khera AV, Chaffin M, Aragam KG, Haas ME, Roselli C, Choi SH, et al. Genome-wide polygenic scores for common diseases identify individuals with risk equivalent to monogenic mutations. Nat Genet. 2018. https://doi. org/10.1038/s41588-018-0183-z.

36. Lambert SA, Abraham G, Inouye M. Towards clinical utility of polygenic risk scores. Hum Mol Genet. 2019;28:R133-42.

37. Janssens ACJW. Validity of polygenic risk scores: are we measuring what we think we are? Hum Mol Genet. 2019. https://doi.org/10.1093/hmg/ddz205.

38. Curtis D. Polygenic risk score for schizophrenia is more strongly associated with ancestry than with schizophrenia. Psychiatr Genet. 2018. https://doi. org/10.1097/YPG.0000000000000206.

39. Tahmasebi $H$, Trajcevski K, Higgins $V$, Adeli K. Influence of ethnicity on population reference values for biochemical markers. Crit Rev Clin Lab Sci. 2018. https://doi.org/10.1080/10408363.2018.1476455.

40. Casey JA, Schwartz BS, Stewart WF, Adler NE. Using electronic health records for population health research: a review of methods and applications. Annu Rev Public Health. 2016. https://doi.org/10.1146/annurevpublhealth-032315-021353.

41. Dennis J, Sealock JM, et al. (2020): Clinical laboratory test-wide association scan of polygenic scores identifies biomarkers of complex disease. GWAS Catalog. https://www.ebi.ac.uk/gwas/. Accessed 20 Nov 2020. 
42. Straub P, Dennis J, Sealock JM, et al (2020): BitBucket. https://bitbucket.org/ straubp_vandy/quality_labs/.

43. Sealock JM, Dennis J, Straub P, et al (2020): BitBucket. https://bitbucket.org/ juliasealock/labwas/.

\section{Publisher's Note}

Springer Nature remains neutral with regard to jurisdictional claims in published maps and institutional affiliations.

Ready to submit your research? Choose BMC and benefit from:

- fast, convenient online submission

- thorough peer review by experienced researchers in your field

- rapid publication on acceptance

- support for research data, including large and complex data types

- gold Open Access which fosters wider collaboration and increased citations

- maximum visibility for your research: over $100 \mathrm{M}$ website views per year

At $\mathrm{BMC}$, research is always in progress. 